\title{
Safety of Early Discharge after Primary Angioplasty
}

\author{
Samra Rehmat and Sajjad Hussain \\ Department of Cardiology, Armed Forces Institute of Cardiology and National Institute of Heart Diseases (AFIC-NIHD), Rawalpindi, \\ Pakistan
}

\begin{abstract}
Objective: To assess the safety of early discharge (less than 48 hours) in ST-segment elevation myocardial infarction (STEMI) patients treated with primary angioplasty (PA).

Study Design: Observational study.

Place and Duration of Study: Armed Forces Institute of Cardiology \& National Institute of Heart Diseases (AFIC-NIHD) From January 2017 to December 2017.

Methodology: All STEMI patients, who underwent primary angioplasty in the above timeframe and then survived to be discharged to home, were included in the study. Patients were divided into two groups based on duration of hospital stay into early and delayed discharge groups (less or more than 48 hours, respectively). The primary outcome measure was all-cause mortality, on day-7, 30,90, and 120-day post-discharge which was ascertained by personal or telephonic follow-up.

Results: During the 12-month study period, 495 patients were successfully discharged to home after PA. Of these, 21 were lost to follow-up. Only the 474 cases followed-up were included in the final analysis. There were 285 patients in early discharge group, and 189 in late discharge group. The mean duration of hospital stay was 64.2 hours. In a multiple logistic regression model, the time of discharge was not affected by age, gender, diabetes mellitus, hypertension, past history of cardiac, or cerebrovascular events, Killip class at presentation, infarct location, angiographic characteristics, procedural details and complications.

Conclusion: There were no differences between the early $(<48 \mathrm{~h})$ and delayed $(>48 \mathrm{~h})$ discharge groups in terms of adverse events. However, this might be due to an overall low event rates in the study.
\end{abstract}

Key Words: ST-elevation myocardial infarction (STEMI), Primary angioplasty, Adverse event, Safety of early discharge after Primary PCI.

How to cite this article: Rehmat S, Hussain S. Safety of Early Discharge after Primary Angioplasty. J Coll Physicians Surg Pak 2020; 30(09):900-905.

\section{INTRODUCTION}

ST-elevationmyocardial infarction (STEMI) patients have historically had a significant degree of long term morbidity, short-term and long term mortality. Although fibrinolysis reduced the mortality and morbidity from STEMI, the effect still had not plateaued; and there was room for improvement. A recent meta-analysis showed that the mortality for STEMI in elderly patients remained 3 to 4 fold higher despite timely fibrinolytic therapy. ${ }^{1}$ Although fibrinolysis helped to a certain degree, the complications such as cardiogenic shock, acute heart failure and mechanical complication remained high. ${ }^{2}$ With the advent of primary angioplasty (PA), the in-hospital complication rates came down and PA is now established to significantly reduce morbidity and mortality of STEMI patients. ${ }^{3}$

Correspondence to: Dr. Sajjad Hussain, Department of Cardiology, Armed Forces Institute of Cardiology and National Institute of Heart Diseases (AFIC-NIHD), The Mall, Rawalpindi, Pakistan

E-mail: sajjad.hussain.cardiologist@gmail.com

Received: January 22, 2020; Revised: August 22, 2020;

Accepted: August 31, 2020

DOI: https://doi.org/10.29271/jcpsp.2020.09.900
The problem of cardiogenic shock-related mortality was ameliorated in a time-related fashion (presentation to balloon time) by PA. ${ }^{4}$ It has been shown in studies that timely reperfusion with PA reduces the mechanical complication rates in STEMI patients. ${ }^{5,6}$ Although a very effective procedure at reducing mortality, PA specially with the trans-radial route comes with its own learning curve that improves with experience. ${ }^{7}$ PA has its own set of procedural complications. ${ }^{8,9}$ These complications and other clinical factors dictate the duration of hospital stay in PA patients. ${ }^{10}$

Optimal duration of hospital stay after PA is not established with a wide range of varying practices among centres worldwide; and remains a grey area with paucity of data. There is currently a lack of consensus to guide the optimal duration of hospital stay afterPA.

The objective of this study was to assess the safety of early discharge in ST-segment elevation myocardial infarction (STEMI) patients treated with primary angioplasty (PA) by evaluating the differences in outcomes in the early and delayed dischargegroups.

\section{METHODOLOGY}

All STEMI patients, who underwent PA between $1^{\text {st }}$ January and 
$31^{\text {st }}$ December 2017 at Armed Forces Institute of Cardiology \& National Institute of Heart Diseases (AFIC-NIHD) and survived to be discharged to home, were included in the study. The operational definition of STEMI diagnosis was based on the third universal definition of myocardial infarction. ${ }^{11}$ PA was done to the infarct related vessel and complete revascularization was left to the discretion of the operator in the event of cardiogenic shock during the procedure. During the hospital stay, all patients had cardiac rhythm and non-invasive hemodynamic monitoring. Before discharge, all patients were advised on the importance of dual antiplatelet therapy, coronary artery disease risk factors, and lifestyle modifications. This was a prospective observational study and designed to be an all-comers real world study in which the patients were discharged based on clinical stability, and clinician discretion.

Patients were divided into 2 groups based on the timing to discharge into an early discharge group: discharged from hospital within 48 hours of PA (Gp1); and a delayed discharge group: those discharged 48 hours after PA (Gp2). Data was collected from the native hospital database. Hospital Management Information System (HMIS). Printed/original handwritten clinical documents/patient notes and records were also consulted, when required. The HMIS reports had the data for the baseline demographics, clinical presentation, pathology lab, imaging, cath procedure details as well as complications. The study began after approval from the Institutional Review Board/Ethical Review Committee (IRB/ERC) of the hospital. The primary outcome measure was all-cause mortality, on days 7 , 30,90 , and 120 -day post-discharge, which was ascertained by personal or telephonic follow-up.

SPSS version 23 (IBM) was used for statistical analysis. Statistical analysis was done by calculating mean and standard deviation for numeric variables. Comparison of means for numeric variables in the two discharge groups was done using the Student t-test. Categorical variables were expressed as frequency percentages between the two groups and were compared using the Pearson Chi-square test/Fisher's Exact test. Kaplan-Meier curves were generated to demonstrate the survival in the early and delayed discharge group; and were compared using the log-rankstatistic. A multiple logistic regression-based model of discharge in early discharge group was generated to see the effect of the following variables at the time of discharge: age, gender, diabetes mellitus, hypertension, previous STEMI or NSTEMI, heart failure with reduced ejection fraction, cerebrovascular events (CVA/TIA), Killip class at presentation, infarct location, post-PCI coronary flow, vascular access site, cardiac rhythm at presentation, multivessel or left main stem disease, and cardiac arrest, or any peri-procedural adverse events. A p-value $<0.05$ (2-sided) was considered statistically significant.

\section{RESULTS}

During the 12-month study period, a total of 495 patients were successfully discharged to home after PA; of these, 21 were lost to follow-up. Only the 474 cases followed up were included in the study. There were $285(60 \%)$ patients in (Gp1), and $189(40 \%)$ in (Gp2). Gender distribution in the two groups was statistically insignificant $(p=0.85)$ with Gp1 having $85.6 \%(n=244)$ and $14.4 \%(n=41)$, and Gp 2 having $86.2 \%(n=163)$; and $13.8 \%$ $(n=26)$ males and females, respectively. Mean age was 59.4 \pm 11.4 and $58.6 \pm 11.0$ years in $\mathrm{Gp1}$ and $\mathrm{Gp2}$ respectively $(p=0.41)$. The frequency of cardiovascular risk factors in the two groups [Gp1-\% (n), Gp2-\% (n), p value] was diabetics [34\% $(n=97), 37.6 \%,(n=71) 0.67]$; hypertensives [54.7\% $(n=156)$, $56.6 \%(n=107), 0.85]$; previous MI [8.1\% $(n=23), 11.6 \%$ $(n=22), 0.36]$; heart failure[1.1\% $(n=3), 0.5 \%(n=1), 0.81]$; previous neurological event $[0.7 \%(n=2), 1.6 \%(n=3), 0.63]$; and previous PCI[7.4\% $(n=21), 10.1 \%(n=19) 0.57]$.

The mean door to balloon times were $49.2 \pm 14$ minutes, and $47.6 \pm 12.2$ minutes for $G p 1$ and $G p 2$, respectively ( $p=0.21)$. Mean ejection fraction was $42.8 \%(S D \pm 6.1), 42.0 \%$ ( $S D \pm 5.9$ ) in $\mathrm{Gp} 1$ and $\mathrm{Gp} 2$, respectively $(\mathrm{p}=0.17)$. The proportion of patients presenting in cardiogenic shock were 3.5\% $(n=10)$ and $5.8 \%$ $(n=11)$ in $G p 1$ and $G p 2$, respectively $(p=0.23)$. Serum creatinine was higher in Gp2 $(1.07 \pm 0.26 \mathrm{mg} / \mathrm{dL})$ compared to $(1.13 \pm 0.39 \mathrm{mg} / \mathrm{dL})$ in $\mathrm{Gp} 1(\mathrm{p}=0.04)$.

The mean duration of hospital stay was $64.2 \pm 61$. 6 hours. Angiographic characteristics and procedure related details are shown in Table I. Vascular access was predominantly radial [95.8\% ( $n=273)$ vs. 94.2\% ( $n=178)$ ] in Gp1 and Gp2, respectively; $p=0.73$ ). There was no statistically significant difference in the angiographic or procedure related characteristics of the two groups. All patients received aspirin and clopidogrel. Majority of patients were discharged on guideline based medical therapy as follows [Gp1, Gp2, p value] - ACE-inhibitors [82.5\% $(n=235), 81.5 \%(n=154), 0.90]$; Angiotensin receptor blockers [3.9\% $(n=11), 4.8 \%(n=9), 0.11]$; bet-blockers [ $63.2 \%$ $(n=180), 54.5 \%(n=103), 0.07]$; and statins [98.2\% $(n=280)$, $96.8 \%(n=183), 0.58]$.

There were no statistically significant differences between the two groups for discharge medications. In a multiple logistic regression model, it was not found any effect of the following variables on the time to discharge: age, gender, diabetes mellitus, hypertension, previous STEMI or NSTEMI, heart failure with reduced ejection fraction, cerebrovascular events (CVA/TIA), Killip class at presentation, infarct location, post PCI TIMI flow, vascular access site, cardiac rhythm at presentation, multivessel or left main stem disease, and cardiac arrest, or any periprocedural adverse events.

The mean duration of follow-up was 17 months in both groups. A total of 19 patients (4\%) died in the study period ( 13 from the early discharge group, and 6 from the delayed discharge group). There was no statistically significant differences in the dead patients between the two groups (Table II). Kaplan-Meier curves for the early and delayed discharge groups are shown in Figure 1. There was no difference in survival between the two groups [Log Rank (Mantel Coxp=0.45)]. 
Table I: Angiographic and procedure related characteristics.

\begin{tabular}{|c|c|c|c|c|c|c|}
\hline \multirow{2}{*}{ Parameter } & & \multicolumn{2}{|c|}{ Gp1 } & \multicolumn{2}{|c|}{ Gp2 } & \multirow{2}{*}{ p-value } \\
\hline & & $\mathbf{n}$ & $\%$ & $\mathbf{n}$ & $\%$ & \\
\hline \multirow{5}{*}{ Infarct related artery } & LAD & 158 & $55.4 \%$ & 101 & $53.4 \%$ & \multirow{5}{*}{0.47} \\
\hline & LCX & 14 & $4.9 \%$ & 11 & $5.8 \%$ & \\
\hline & RCA & 107 & $37.5 \%$ & 77 & $40.7 \%$ & \\
\hline & Diagonal & 1 & $0.4 \%$ & 0 & $0.0 \%$ & \\
\hline & OM & 2 & $0.7 \%$ & 0 & $0.0 \%$ & \\
\hline \multirow{3}{*}{ Multivessel disease } & 1 & 96 & $33.7 \%$ & 67 & $35.4 \%$ & \multirow{3}{*}{0.91} \\
\hline & 2 & 96 & $33.7 \%$ & 61 & $32.3 \%$ & \\
\hline & 3 & 93 & $32.6 \%$ & 61 & $32.3 \%$ & \\
\hline \multirow{2}{*}{ LMS disease } & Yes & 34 & $11.9 \%$ & 13 & $6.9 \%$ & \multirow{2}{*}{0.07} \\
\hline & No & 251 & $88.1 \%$ & 176 & $93.1 \%$ & \\
\hline \multirow{2}{*}{ Bifrucation Lesion } & Yes & 46 & $16.1 \%$ & 28 & $14.8 \%$ & \multirow{2}{*}{0.69} \\
\hline & No & 239 & $83.9 \%$ & 161 & $85.2 \%$ & \\
\hline \multirow{3}{*}{ IRA Post TIMI 3 Flow } & Yes & 280 & $98.2 \%$ & 188 & $99.5 \%$ & \multirow{3}{*}{0.41} \\
\hline & No & 5 & $1.8 \%$ & 1 & $0.5 \%$ & \\
\hline & Unknown & 0 & $0.0 \%$ & 0 & $0.0 \%$ & \\
\hline \multirow{2}{*}{ Balloon Pre Dil } & Yes & 257 & $90.2 \%$ & 170 & $89.9 \%$ & \multirow{2}{*}{0.94} \\
\hline & No & 28 & $9.8 \%$ & 19 & $10.1 \%$ & \\
\hline \multirow{3}{*}{ Use of Atherothrombectomy } & Yes & 15 & $5.3 \%$ & 7 & $3.7 \%$ & \multirow{3}{*}{0.52} \\
\hline & No & 269 & $94.4 \%$ & 182 & $96.3 \%$ & \\
\hline & Unknown & 1 & $0.4 \%$ & 0 & $0.0 \%$ & \\
\hline \multirow{3}{*}{ Stent used } & DES & 280 & $98.2 \%$ & 185 & $97.9 \%$ & \multirow{3}{*}{0.86} \\
\hline & BMS & 2 & $0.7 \%$ & 1 & $0.5 \%$ & \\
\hline & POBA only & 3 & $1.1 \%$ & 3 & $1.6 \%$ & \\
\hline \multirow{2}{*}{ Use of Gpll-b/ Illa inhibitors } & Yes & 285 & $100.0 \%$ & 187 & $98.9 \%$ & \multirow{2}{*}{0.16} \\
\hline & No & 0 & $0.0 \%$ & 2 & $1.1 \%$ & \\
\hline \multirow{2}{*}{ IABP } & Yes & 2 & $0.7 \%$ & 3 & $1.6 \%$ & \multirow{2}{*}{0.39} \\
\hline & No & 283 & $99.3 \%$ & 186 & $98.4 \%$ & \\
\hline
\end{tabular}

Table II: Differences in-between the dead patients in the two groups.

\begin{tabular}{|c|c|c|c|c|}
\hline & Groups & $\mathbf{N}$ & Mean \pm SD & $\mathbf{p}$ \\
\hline \multirow{2}{*}{ Age } & Gp1 & 13 & $67.69( \pm 10.66)$ & \multirow[t]{2}{*}{0.95} \\
\hline & Gp2 & 6 & $67.33( \pm 12.83)$ & \\
\hline \multirow{2}{*}{ DBT } & Gp1 & 13 & $46.92( \pm 4.80)$ & \multirow[t]{2}{*}{0.81} \\
\hline & Gp2 & 6 & $46.33( \pm 4.97)$ & \\
\hline \multirow[b]{2}{*}{ SBP } & Gp1 & 13 & $132.08( \pm 29.64)$ & \multirow[t]{2}{*}{0.68} \\
\hline & Gp2 & 6 & $125.83(S D \pm 30.22)$ & \\
\hline \multirow[b]{2}{*}{ DBP } & Gp1 & 13 & $73.69(S D \pm 22.58)$ & \multirow[t]{2}{*}{0.81} \\
\hline & Gp2 & 6 & $76.17(\mathrm{SD} \pm 14.62)$ & \\
\hline \multirow{2}{*}{ Haemoglobin } & Gp1 & 13 & $13.51( \pm 0.70)$ & \multirow[t]{2}{*}{0.72} \\
\hline & Gp2 & 6 & $13.40( \pm 0.0)$ & \\
\hline \multirow{2}{*}{ Serum Creatinine } & Gp1 & 13 & $1.26( \pm 0.33)$ & \multirow[t]{2}{*}{0.23} \\
\hline & Gp2 & 6 & $1.09( \pm 0.0)$ & \\
\hline \multirow[b]{2}{*}{ Blood Glucose } & Gp1 & 13 & $165.69( \pm 127.35)$ & \multirow[t]{2}{*}{0.63} \\
\hline & Gp2 & 6 & $140.0( \pm 0.0)$ & \\
\hline \multirow[b]{2}{*}{ EF } & Gp1 & 13 & $38.85( \pm 5.06)$ & \multirow[t]{2}{*}{0.88} \\
\hline & Gp2 & 6 & $39.17( \pm 2.04)$ & \\
\hline
\end{tabular}

\section{DISCUSSION}

The authors sought to find any difference between early and delayed discharge groups in patients undergoing PA. The main finding of this study is the lack of a significant difference in the mortality between early and delayed discharge from the hospital. The possible reason may be that the overall mortality in this PA cohort was $4 \%$. Only one patient had died within the first seven days; six by 30 days; 14 by 90 days and 19 by 180 days. There were no further deaths in either group. Thirteen (13) of these patients were discharged in less than 48 hours and six were discharged 
after 48 hours; the mortality actually being higher in the early discharge group. In this study, the two groups were not significantly different from each other clinically, angiographically and in the conduct of the primary $\mathrm{PCl}$ procedure (Table I) or with respect to treatment on discharge. It appears that the decision to discharge the patient was clearly a clinical one, based on hemodynamic and ECG monitoring. Multiple logistic regression model did not identify any of the studied variables to influence the discharge decision. The clinical characteristics of the dead patients in both groups (Table II) were not significantly different regarding age, door to balloon time, systolic and diastolic BP on presentation, hemoglobin, serum creatinine, blood glucose and ejection fraction.

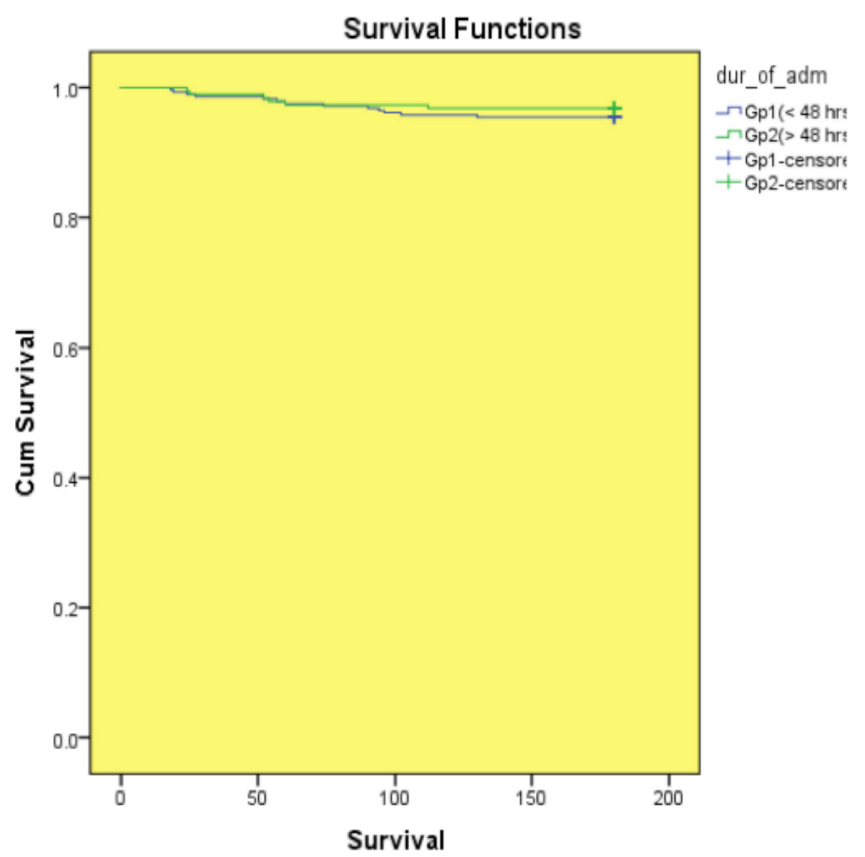

Figure 1: Kaplan-Meier curves for the early and delayed discharge groups.

$\mathrm{PCl}$ has progressed significantly over the last decade, in moving from femoral access to radial access, reduction in the size of catheters and the availability of adjunctive tools to handle more complex lesion subsets. With these developments, emerges the reality that complications are decreasing over time and, hence, no longer require prolonged hospital stay except in select patients. But because post-PCl complications carry a significant degree of risk, so physicians tend to err on the side of caution and prolong hospital stay. The results from the Champion Phoenix trial showed that almost all adverse events occurred within six hours from the index procedure, and plateaued out after 42 hours. ${ }^{12}$ The median duration of stay in this study was 46.5 hours, and mean 64.2 hours. These results have been already been demonstrated by the earlier STRIDE and DISCHARGE studies. ${ }^{13,14}$ The DISCHARGE investigators concluded that the presence of high risk factors per se was not an absolute indication for overnight admission after angioplasty. ${ }^{15}$ However, it is a well-known fact that high risk, acute coronary syndrome patients with complications tend to have longer hospital stay. ${ }^{15-17}$ A useful expert analysis of the current evidence on the length of hospital stay after PA has suggested that there is no benefit in terms of safety in keeping a patient in hospital after an uncomplicated three days post-PCl. ${ }^{18}$

Hospital stay after PA in STEMI patients incurs significant costs and it is useful to identify these patients for early discharge. ${ }^{12}$ In the thrombolysed patients, safety of early discharge in low risk patients has already been demonstrated. ${ }^{19}$ Despite extensive research, there is still a wide regional variation in duration of hospital stay patterns in acute myocardial infarction in different parts of the world; and there doesn't seem to be a consensus about the optimal timing of discharge in PA patients. In one major study, the room for more economical, efficient and safe discharges (for low risk patients) existed in all the countries investigated; but was especially evident in Belgium, France, Germany, Spain, and Poland. ${ }^{14}$ With growing experience, the overall duration of hospital admission in STEMI patients has decreased with time. ${ }^{15}$ The safety of short hospital stay in selected low risk patients has been demonstrated in different studies with low short and long term mortality. There is abundant data to support that lack of difference in adverse outcomes in low risk patients who have been discharged early or at a delayed date. ${ }^{15-17}$ The guidelines now acknowledge a change in the definition of early discharge; in 2012 it was $72 \mathrm{~h}$, and in 2017 it is $48-72$ h. ${ }^{20}$ The key messages in the guidelines state that "Early ambulation and early discharge are the best option in uncomplicated patients". ${ }^{20}$ In the era of PA, it seems intuitive that with the reduction in mortality and complications ${ }^{3}$ there should be a trend towards earlier discharge. In addition, the reperfusion and patent artery rates are also higher with PA than with fibrinolysis. The cost reduction with early hospital discharge is also substantial. The PAMI II investigators showed that early identification of low risk patients allowed early discharge and substantial cost reduction. ${ }^{21}$ Other studies have documented similar cost reductions after early hospital discharge following PA. ${ }^{22}$

Although it has been demonstrated in some studies that it is safe to discharge low risk patients early, to date there have been no such studies in an all comers Pakistani population. The logistic regression analysis in this study failed to identify any significant difference in variables between the early and delayed discharge groups, demonstrating the physician bias in keeping the patients in hospital for a longer duration after PA. This has significant cost implications for the patients and public sector hospitals. Although comparable with the contemporary data, we feel that the event rates have been low to provide a robust analysis. A larger future study with more event rates will likely answer these questions. 


\section{CONCLUSION}

This study results found no differences between the early $(<48 \mathrm{~h})$ and delayed ( $>48 \mathrm{~h})$ discharge groups in terms of adverse events. However, the authors feel that this is because of the low event rates in this study, and further study is warranted in this area.

\section{ETHICAL APPROVAL:}

The study began after approval from the Institutional Review Board/Ethical Review Committee (IRB/ERC) of the Hospital (AFIC-NIHD).

\section{PATIENTS' CONSENT:}

Since the patient data was anonymised and no individual patient could be identified, no specific informed consent was obtained for use of patient data for research.

\section{CONFLICT OF INTEREST:}

The authors declared no conflict of interest.

\section{AUTHORS' CONTRIBUTION:}

SR: Contributed towards initial synopsis writing and IERB approval, data collection and manuscript writing.

$\mathrm{SH}$ : Contributed towards data entry in SPSS, data analysis, manuscript writing and revisions.

\section{REFERENCES}

1. Ahmed S, Antman EM, Murphy SA, Giugliano RP, Cannon CP, White $\mathrm{H}$, et al. Poor outcomes after fibrinolytic therapy for ST-segment elevation myocardial infarction: Impact of age (a meta-analysis of a decade of trials). J Thromb Thrombolysis 2006; 21(2):119-29. doi: 10.1007/s11239006-5485-9.

2. Wang $W$ and Cheung A. Mechanical Complications of Acute Myocardial Infraction. In: TJ Watson, PJL Ong and JE Tcheng, eds. Primary Angioplasty: A practical guide Singapore 2018; 275-87.

3. Keeley EC, Boura JA and Grines CL. Primary angioplasty versus intravenous thrombolytic therapy for acute myocardial infarction: A quantitative review of 23 randomised trials. Lancet 2003; 361(9351):13-20. doi: 10.1016/ S0140-6736(03)12113-7.

4. Scholz KH, Maier SKG, Maier LS, Lengenfelder B, Jacobshagen $\mathrm{C}$, Jung J, et al. Impact of treatment delay on mortality in ST-segment elevation myocardial infarction (STEMI) patients presenting with and without haemodynamic instability: Results from the German prospective, multicentre FITT-STEMI trial. Eur Heart J 2018; 39(13):1065-1074. doi: 10.1093/eurheartj/ehy004.

5. Nakatani $D$, Sato $H$, Kinjo K, Mizuno $H$, Hishida E, Hirayama $A$, et al. Acute coronary insufficiency study G. Effect of successful late reperfusion by primary coronary angioplasty on mechanical complications of acute myocardial infarction. Am J Cardiol 2003; 92(7):785-8. doi: 10.1016/s00029149(03)00883-x.

6. Navarro-Cuartero J, Cordoba-Soriano JG, Jimenez-Mazuecos J, Gutierrez-Diez A, Gallardo-Lopez A and Melehi D. Incidence and prognosis of mechanical complications of STEMI after primary angioplasty: Data from a single-centre registry of an infarction code program. Rev Esp Cardiol (Engl Ed) 2016; 69(9):874-6. doi: 10.1016/j.rec.2016. 04.032

7. Hess CN, Peterson ED, Neely ML, Dai D, Hillegass WB, Krucoff MW, et al. The learning curve for transradial percutaneous coronary intervention among operators in the United States: A study from the national cardiovascular data registry. Circulation 2014; 129(22):2277-86. doi: 10.1161/CIRCULATIONAHA.113.006356.

8. Wijesinghe N, Nunn C, Sebastian C, Heald S, McAlister $\mathrm{H}$ and Devlin G. Complications of primary angioplasty in myocardial infarction (PAMI) in a real-world cardiac catheterisation laboratory: 8-year experience. Heart, Lung and Circulation 2008; 17(3):S175. DOI:http://doi.org/ 10.1016/j.hlC.2008.05.416

9. Anderson HV, Shaw RE, Brindis RG, Hewitt K, Krone RJ, Block $\mathrm{PC}$, et al. A contemporary overview of percutaneous coronary interventions. The American college of cardiology-national cardiovascular data registry (ACC-NCDR). J Am Coll Cardiol 2002; 39(7):1096-103. doi: 10.1016/s0735-1097 (02)01733-3.

10. Seto AH, Shroff A, Abu-Fadel M, Blankenship JC, Boudoulas $K D$, Cigarroa JE, et al. Length of stay following percutaneous coronary intervention: An expert consensus document update from the society for cardiovascular angiography and interventions. Catheterisation and cardiovascular interventions: Official J Society Cardiac Angiography Interventions 2018; 92(4): 717-31. doi.org/ 10.1002/ ccd.27637.

11. Thygesen K, Alpert JS, Jaffe AS, Simoons ML, Chaitman BR, White $H D$, et al. Third universal definition of myocardial infarction. J Am Coll Cardiol 2012; 60:1581-98.

12. Kinjo K, Sato H, Sakata $Y$, Nakatani D, Mizuno H, Shimizu M, et al. Acute coronary insufficiency study g. identification of uncomplicated patients with acute myocardial infarction undergoing percutaneous coronary intervention: Are these patients suitable for early discharge? Circ J 2005; 69: 1163-9. doi.org/10.1253/circj.69.1163.

13. Jabara R, Gadesam R, Pendyala L, Chronos N, Crisco LV, King SB, et al. Ambulatory discharge after transradial coronary intervention: Preliminary US single-center experience (Same-day transradial intervention and discharge evaluation, the stride study). Am Heart J 2008; 156 (6):1141-6. doi: 10.1016/j.ahj.2008.07.018.:1141-6.

14. Kaul P, Newby LK, Fu Y, Mark DB, Califf RM, Topol EJ, et al. International differences in evolution of early discharge after acute myocardial infarction. Lancet 2004; 363 (9408):511-7. doi: 10.1016/S0140-6736(04)15536-0.

15. Tran HV, Lessard D, Tisminetzky MS, Yarzebski J, Granillo EA, Gore JM, et al. Trends in length of hospital stay and the impact on prognosis of early discharge after a first uncomplicated acute myocardial infarction. Am J Cardiol 2018; 121(4):397-402. doi: 10.1016/j.amjcard.2017. 11.001.

16. Melberg T, Jorgensen M, Orn S, Solli T, Edland U and Dickstein K. Safety and health status following early discharge in patients with acute myocardial infarction treated with primary PCI: A randomised trial. Eur J Prev Cardiol 2015; 22(11):1427-34. doi: 10.1177/20474873 14559276.

17. Azzalini L, Sole E, Sans J, Vila M, Duran A, Gil-Alonso D, et al. Feasibility and safety of an early discharge strategy after 
low-risk acute myocardial infarction treated with primary percutaneous coronary intervention: The EDAMI pilot trial. Cardiology 2015; 130(2):120-9. doi: 10.1159/000368890.

18. Elliott Groves MIV. Length of stay after PCI Latest In Cardiology JACC 2019.

19. Ross RS. Early discharge after heart attacks and the efficient use of hospitals. N Engl J Med 1978; 298(5):275-7. DOI: 10.1056/NEJM197802022980510.

20. Ibanez B, James S, Agewall S, Antunes MJ, Bucciarelli-Ducci C, Bueno H, et al. 2017 ESC Guidelines for the management of acute myocardial infarction in patients presenting with ST-segment elevation: The task force for the management of acute myocardial infarction in patients presenting with ST-segment elevation of the European society of cardiology (ESC). European Heart J 2017; 39(2):119-177. doi: 10.1093/ eurheartj/ehx393.

21. Grines CL, Marsalese DL, Brodie B, Griffin J, Donohue B, Costantini CR, et al. Safety and cost-effectiveness of early discharge after primary angioplasty in low risk patients with acute myocardial infarction. PAMI-II Investigators. Primary angioplasty in myocardial infarction. J Am Coll Cardiol 1998; 31(5):967-72. doi: 10.1016/s0735-1097(98) 00031-x.

22. Eastwood GL. Cost effectiveness of early discharge after uncomplicated acute myocardial infarction. $N$ Engl J Med 2000; 343(9):658. doi: 10.1056/NEJM200008313430912. 